\title{
A Miniaturised Piezoresistive Multi-Component Force Sensor for Minimally Invasive Surgery
}

\author{
Rausch, Jacqueline, Werthschützky, Roland \\ Institut für Elektromechanische Konstruktionen, Technische Universität Darmstadt \\ Merckstr. 25 \\ 64287 Darmstadt
}

\begin{abstract}
Conventional tools used in minimally invasive abdominal surgery (MIS) suffer from workspace restrictions and loss of haptic perception of the manipulated tissue. Operations are more complicated, expert surgeons are needed and longer operation time is the consequence. On this, a novel hand-held instrument with a force feedback system for gentle manipulation of soft tissue is being developed [1]. Driven by integrated piezoelectric actuators an operation platform with four additional degrees of freedom carries manipulators and positions them inside the surgical area. To assist the surgeon during the operation the relating forces between soft tissue and tool are measured with a force sensor. Typically the force vector consists of three force components $F_{i}$, each one with an absolute value of $\left|F_{i}\right| \leq 5 \mathrm{~N}$ [2]. An active control element is feeding back the haptic information to the surgeons' hand.

In this paper we report of the progress of a miniaturised multi-component force sensor. It will be integrated in the tool tip of the operating platform and detect at minimum three force components (shear and normal forces). Following the requirements of humans' haptic perception the sensor detects both static and dynamic forces up to $1000 \mathrm{~Hz}$. According to forces arising in typical tasks especially during liver surgery forces up to $5 \mathrm{~N}$ can be measured at the tool tip.
\end{abstract}

\section{Introduction}

Minimally invasive abdominal surgery (MIS) has become a state-of-the-art technique in surgery over the past decades. Several standard procedures and suitable instruments have been developed [3]. The interventions cover a wide range of possible treatments e.g. in thorastic surgery, brain surgery and abdominal surgery. The main advantage of minimally invasive techniques is shorter patient recovery time in comparison to open surgery due to the reduction of tissue trauma and risks of postoperative infection. Though many interventions like cholecystectomy (removal of gall bladder) for example are already standard procedures in laparoscopic surgery. The main disadvantage of conventional laparoscopes is the loss of intracorporeal workspace, because the number of degress of freedom is limited to four. Additionally, the haptic feedback is deminished due to friction in trocars and restoring forces of the abdominal wall. Thus, the surgeon is loosing one of his most important senses - the sense of haptic perception. It disables him to feel tissue hardness, evaluate tissue properties and anatomical structures. Furthermore, it inhibits him to commit appropriate force controlled actions for safe tissue manipulation.

Several approaches have been suggested to implement force measurement for haptic feedback in laparoscopic tools $[4,5]$. On the one hand conventional laparoscopic tools are equiped with force sensing elements usually strain gauges (for example [6,7]), on the other hand laparoscopic tools have been modified or even redesigned integrating a force sensor $[7,8,9,11,12]$. The itemised examples vary in sensing principle, dimensions and location of the force sensor. ROSEN et al. at the University of Washington [7] integrate sensing elements outside of the abdominal cavity (see Fig. 1(b)). They motorised a laparoscopic grasper with DC motors controlling Bowden cables and evaluated the acting forces integrating conventional strain gauges within the driving mechanism. Because of the extracorporeal location the sensors have not to be sterilisable. Forces up to $60 \mathrm{~N}$ have been measured. According to the distance between sensor and interaction point, the friction of every joint and the backlash of the kinematics itself decrease the accuracy. Furthermore the dynamics are limited because the kinematics act like a mechanical low-pass-filter. BERKELMAN et al. [8] developed a microsurgical tip force sensing instrument (see Fig. 1(a)). The piezoresisitve tri-axial force sensor is integrated into the hand-held and has an outer diameter of $12.5 \mathrm{~mm}$ and a height of $15 \mathrm{~mm}$. Nominal load of $+/-1 \mathrm{~N}$ at a resolution of $0.5 \mathrm{mN}$ are detectable. Other research groups measure forces at the distal end of an instrument. Either being sterilisable or a sterile one-way product and thus miniaturised ${ }^{1}$, the requirements on dimensions of these sensing elements and their packaging increase. At the Katholieke Universiteit

\footnotetext{
${ }^{1}$ diameters $\leq 10 \mathrm{~mm}$ are state of the art for minimally invasive surgery tools
} 
Leuven PEIRS et al. [9] developed a optical tri-axial sensor with a diameter of $5 \mathrm{~mm}$ (see Fig. 1(d)). Forces up to $2.5 \mathrm{~N}$ can be detected with a resolution of $40 \mathrm{mN}$. The dynamics of the sensor and the ability of sterilisation are unknown. Apart from the optical sensor resistive measurement principle is dominating. At the Institute of Robotics and Mechatronics at German Aerospace Center (DLR) [11], a complex forcetorque-sensor has been integrated into a laparoscopic grasper (see Fig. 1(c)). On this a hexapodian structure (also known as Steward platform) fabricated from AlCuMg-alloy and equiped with conventional strain gauges is mounted between shaft and grasping shaws. The sensor has a diameter of $10 \mathrm{~mm}$. Forces up to $20 \mathrm{~N}$ with a resolution of $250 \mathrm{mN}$ for axial force components and $50 \mathrm{mN}$ for lateral ones can be detected. The dynamics and the ability to sterilisation are unknown. The sensitivity of the sensor is limited by the high stiffness of the structure and the low gauge factor of about 2 of conventional strain gauges.

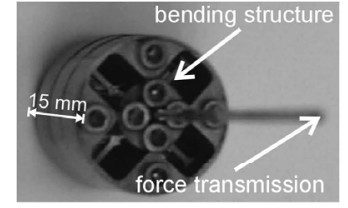

(a) Tri-axial force sensor (diameter of $12.5 \mathrm{~mm}$ ) published by Berkelman et al. [8]

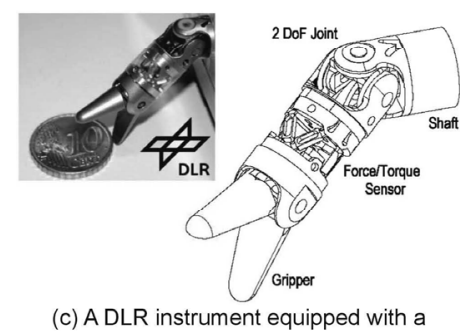

six-axis force-torque sensor [11]

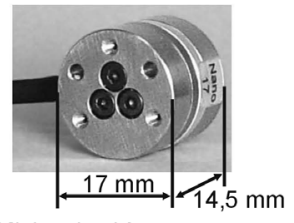

(b) Miniaturised force torque senso used in [7] manufactured by ATI
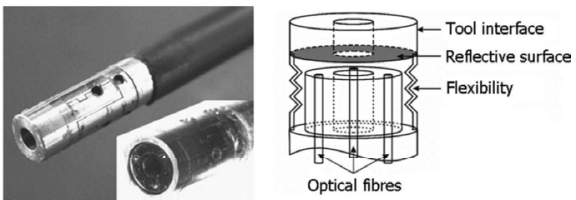

(d) An optical force sensor using three optica fibres to perform force measurement [9]

Fig. 1. Force measurement in minimally invasive tools.

Due to huge dimensions, low nominal load and frequency range or low sensitivity, the presented examples are not suited for being integrated into our instrument INKOMAN. In this paper we report of the progress of piezoresistive force sensors for gentle liver surgery. The tri-axial sensor is also integrated at the distal end of a novel laparoscopic tool. The forces occuring beween instrument and tissue deform the tool tip. To achieve a higher sensitivity silicon strain gauges $(k \approx 80)$ are used in our work. The sensing principle is the piezoresistive effect and thus the resistivity change is a function of resultant stress distribution inside the sensing element [13].

The paper is organised as follows:

Section two reports the development of the haptic sensor for soft tissue manipulation, particularly the identification of requirements, design of deformation and sensing elements. First functional models of the sensor are presented and discussed. Section three outlines the sensor development and gives an outlook on future work.

\section{Development of the Piezoresistive Force Sensor}

Based on an analysis of interesting interactions in liver surgery and important parameters of humans haptic perception the requirements for the haptic sensor have been collected. Following these specifications the development of the sensors is done.

\subsection{Requirements}

The requirements on the sensor arise from the application itself and the position of measurement. In our case the instrument interacts with soft liver tissue. The interesting surgery tasks are pulling and pushing tissue, cutting tissue with laser scalpels or electrocauters and rupture of tissue. In co-operation with medical experts typical minimally invasive tasks were performed on ex-vivo porcine and bovine liver tissue. The resultant forces has been measured [2]. The following tasks have been accomplished: blunt dissection of the gallbladder, resection of liver tissue with a laser-scalpel and shifting tissue. In order to synchronise the measured values to the accomplished tasks, interactions between force measuring endoscope and tissue were recorded with a video camera. Absolute values of force up to $5 \mathrm{~N}$ occured, thus the nominal force value of each component should take this value at minimum. The sensor has to be overload-protected up to $20 \mathrm{~N}$ in case of interacting with stiffer materials like e.g. other instruments. In our application a novel laparoscopic instrument is being developed which carries and positions manipulation 
tools in the abdominal cavity. The force transmission occurs at the manipulation tool itself ${ }^{2}$, which is attached at the positioning platform. Thus, the platform is used as deformation element and the strain sensing elements are bonded on it. Depending on the dimensions of the attached instrument ${ }^{3}$ and the maximal outer diameter, the available space is a hollow cylinder with an inner diameter of about $4 \mathrm{~mm}$ and an outer diameter smaller than $10 \mathrm{~mm}$. The maximal height is $10 \mathrm{~mm}$ according to the instruments height.

For a realistic haptic feedback the dynamics of the sensor are the crucial factor. Thus both static and dynamic forces up to $400 \mathrm{~Hz}$ at minimum ${ }^{4}$ are required. Unfortunatly, the upper cut-off frequency wasn't detectable during the measurement due to the high noise of the used evaluation unit. Only the interaction frequency of up to $10 \mathrm{~Hz}$ has been measured. The resolution results from the physiological value Just Notable Difference (JND) where $10 \%$ of the acting force is detected. Hence, a resolution of $\leq 100 \mathrm{mN}$ is claimed. ${ }^{5}$ The measurement uncertainty should take values smaller than $10 \%$ at maximum.

\subsection{A First Functional Model - 2-Axial Force Sensor}

Due to the described requirements a functional model measuring lateral force components has been developed. Because of its well known mechanical behaviour a beam bender was chosen as deformation element. Its dimensions result from the integration into the tool tip and the required relative resistivity change of the silicon strain gauge (2\% at nominal load [15]). Accordingly, the resulting nominal strain has to be nearly $2.5 \cdot 10^{-4}$ at nominal load (see Fig. 2(a)). Using BERNOULLI's bending theory the dimensions take a length of $9 \mathrm{~mm}$, a height of $2 \mathrm{~mm}$ and a width of $1.4 \mathrm{~mm}$. In a Finite Elements Analysis (FEA) using the software ProMechanica the bending behaviour has been analysed. Forces affecting the tip laterally (y direction) are causing a strain gradient in the same direction as the acting force. Forces affecting the tip vertically ( $x$ direction) are leading to homogenously distributed strain. Fig. 2(b) shows the realised assembly. To detect the described strain distribution depending on the acting force component a crosslike assembly of the strain sensitive resistors is needed. Silicon pressure sensors occupy the piezoresistive areas in the required assembly, but the geometry of the sensor is not suitable for detecting the strain distribution. Thus the silicon pressure sensor is modified: On one hand for being sensitive to bending strain, on the other hand to obtain four separate piezoresistive areas for being able to interconnect them in the appropriate way. To decouple the adhesive area from the measuring position and hence the position of the resistors the bridge-like geometry is chosen for the silicon element (see Fig. 2(a)). One silicon element has a height of $390 \mu \mathrm{m}$ (diaphragm thickness nearly $40 \mu \mathrm{m}$ ), a length of $2 \mathrm{~mm}$ and a width of about $1 \mathrm{~mm}$. It can be used for detecting lateral forces. For the realisation of the force sensor two modified silicon elements are mounted on top and bottom of the cantilever for measuring the surface strain. The used adhesive is a two component epoxy. For contacting the sensing elements wire bonds have been applied. The resulting resistance change of the piezoresistive areas in the gauge are evaluated by a current-fed Wheatstone full bridge for each force component [1]. Fig. 2(c) shows the interconnection of the resistors used for detecting forces in y direction. For detecting forces in $y$-direction, $R_{y 1}$ and $R_{y 2}$ located at the top side of the element are connected with $R_{y 3}$ and $R_{y 4}$ at the bottom side. $R_{x 1}$, $\mathrm{R}_{\mathrm{x} 2}, \mathrm{R}_{\mathrm{x} 3}$ and $\mathrm{R}_{\mathrm{x} 4}$ are used for the $\mathrm{x}$-direction accordingly.

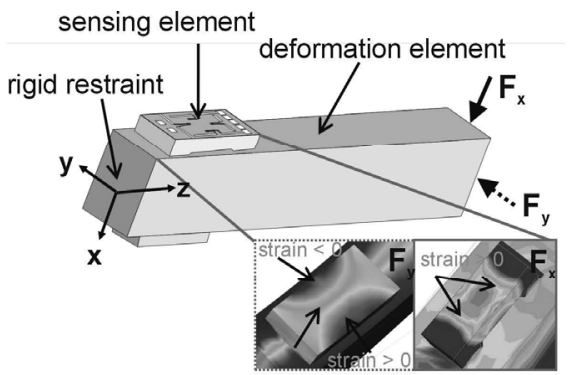

(a) Resulting strain distribution caused by lateral forces

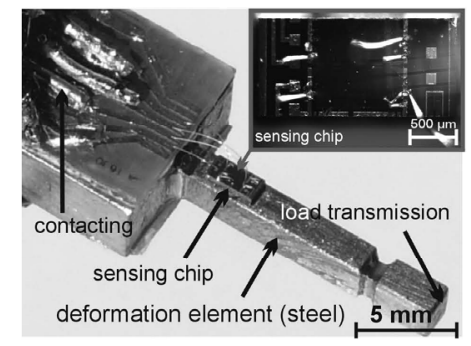

(b) Realised two-component force sensor

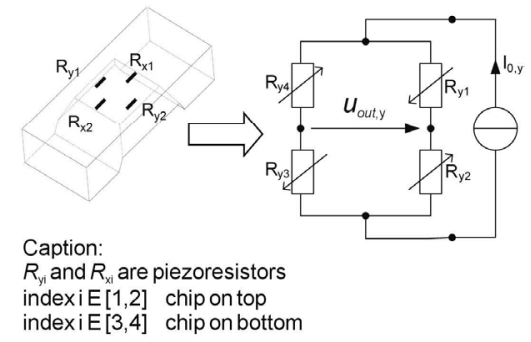

(c) Data aquisition

Fig. 2. First functional model for detecting lateral forces acting to the tool tip

The resulting characteristics of the realised sensor are summarised in Table 1. The measured sensitivity and the resolution respectively differ from the simulated values maybe due to the unaccounted epoxy layer for the simulated setup among others.

\footnotetext{
${ }^{2}$ Apart form using non-contact tools like e.g. laser scalpels. At this the force transmission occurs at the deformation

element.
3 In our application a instrument with a diameter of $3.8 \mathrm{~mm}$.

${ }^{4}$ The maximum sensitivity is around $340 \mathrm{~Hz}$. An upper cut-off-frequency of $1 \mathrm{kHz}$ is desirable to realise a more realistic haptic feedback.

${ }^{5}$ The lowest interaction force of about $1.1 \mathrm{~N}$ occurs while cutting liver tissue with a laser scalpel [2].
} 
Tab. 1. Measured characteristics of the two-component force sensor

\begin{tabular}{lcc} 
Parameter & Specification & Measured Value \\
\hline Nominal force & $\geq 5 \mathrm{~N}$ & $5 \mathrm{~N}$ \\
Sensitivity $u_{\text {out }}\left(I_{0} \cdot R_{0}\right) @ 1 \mathrm{~N}$ & $0.4 \%$ & $\approx 0.3 \%$ \\
Resolution $\Delta F$ & $0.01 \mathrm{~N}$ & $0.02 \mathrm{~N}$ \\
Hysteresis error $f_{\mathrm{H}}$ & $\leq 10 \%$ & $\leq 2 \%$ \\
Nonlinearity $t_{\mathrm{L}}$ & $\leq 5 \%$ & $\leq 1 \%$
\end{tabular}

\subsection{Design of the Tri-Axial Force Sensor}

In the following a redesign of the force sensor is proposed. On the one hand novel silicon strain gauges are being developed [9] on the other hand the topology of the deformation element is being redesigned for measuring three force components at the tool tip of a laparoscopic instrument. The mechanical behaviour according to the geometry of the silicon element is being analysed. Hence, the backlash on the bending behaviour of the deformation element is analytically calculated and the stress distribution in the sensing element itself is simulated. Based on this, the piezoresistive areas are arranged and finally the data acquisition is defined.

\subsubsection{Deformation Element}

The interactions executed by the sensorised tool during surgery determine the required numbers of force components and torques. At minimum three force components have to be detected. In case of grasping tissue also the detection of torques is necessary. Concerning i.a. monolithic fabrication the topology shown in Fig. 3 (b) is chosen. The bending elements can be dimensioned for by considering the necessary nominal strain for the sensing element ${ }^{6}$. Using equations 1 and 2 resulting from CASTIGLIANOS theory the dimensions are determined $[5,13]$.

$$
\begin{aligned}
& \varepsilon_{T}=\frac{F \cdot b}{2 \cdot E I} \cdot\left(\frac{b \cdot G \cdot I_{t}+2 \cdot a \cdot E I}{b \cdot G \cdot I_{t}+a \cdot E I}\right) \cdot \frac{h}{2} \quad, \text { where } I=\frac{b \cdot h^{3}}{12}, \quad I_{t}=\sqrt{2} \cdot h^{4} \\
& \varepsilon_{R}=\left(\frac{F \cdot a^{2}}{2} \cdot \frac{G \cdot I_{t}}{a \cdot G \cdot I_{t}+a \cdot G \cdot E I}+F \cdot a\right) \cdot \frac{h}{2 \cdot E I}
\end{aligned}
$$

$\varepsilon_{\mathrm{T}}$ is the resulting bending strain at the force transmission point and $\varepsilon_{\mathrm{R}}$ at the rigid restraint, $F$ the acting force, $E$ the Youngs Modulus and $G$ the Shear Modulus of the bending material ${ }^{7}, l, a, b$ and $h$ parameters of the bending element shown in Fig. 3(a) and the geometrical moments of inertia $l$.

Width and height of the bending element are fixed parameters due to the dimension of the sensing element each with a value of $1 \mathrm{~mm}$ and so are the geometrical moments of inertia. The values of $a$ and $b$ are calculated using Matlab. The fitting values are shown in Fig. 3. Due to cylindrical topology and limited available space curved sections are used for the bending elements (see Fig. 3(b)). Equations 1 refer to strain bending elements and the calculated values are only an approximation. Therefore the values calculated in Matlab have been verified in a FEA using ProMechanica. Fig. 3(b) shows one of the designed deformation elements including three bending elements. Determining compliance $n$ and mass $m$ of the deformation element the theoretical resonance frequency of the realised force sensor is at about $7 \mathrm{kHz}$.

\subsubsection{Sensing Element}

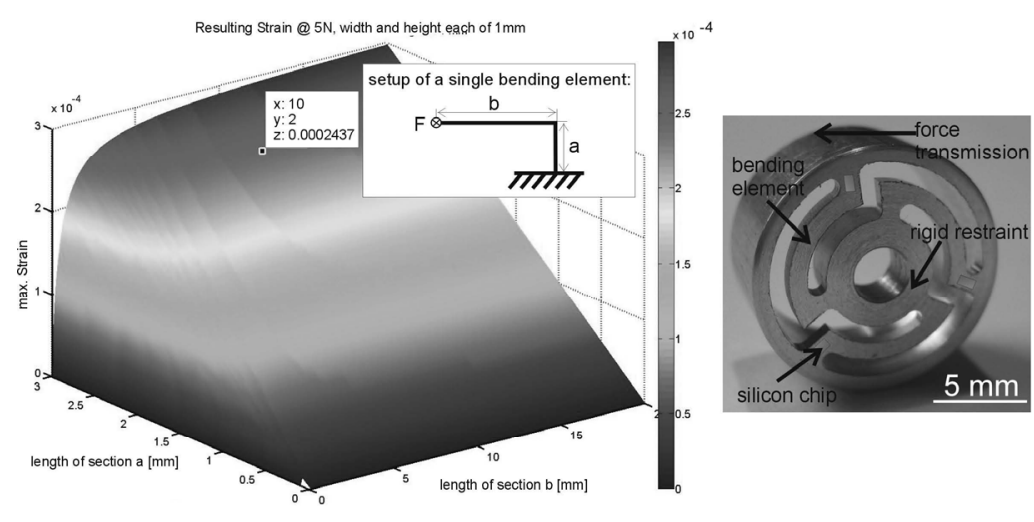

(a) Resulting strain at the force transmission point ploted against lengths of $a$ and $b$

(b) Deformation element of the tri-axial sensor. In the center a tool will be integrated.

Fig. 3. Dimensioning of deformation elements for miniaturised triaxial force sensors

The stress $(\sigma)$ distribution in the silicon element has been analysed to arrange the strain $(\varepsilon)$ sensing areas in the right way. Due to the piezoresistive effect the resistivity change is depending on the position and orientation of the resistive areas. Hence, direction and orientation of the current density $J$ is related to the

\footnotetext{
${ }_{7}^{6}$ About $250 \mu \mathrm{m} / \mathrm{m}$ as noted in paragraph 2.2 .

${ }^{7}$ In a first realisation aluminium is used with a Youngs Modulus of about $73.000 \mathrm{~N} / \mathrm{mm}^{2}$ and Shear Modulus G about $28.000 \mathrm{~N} / \mathrm{mm}^{2}$.
} 
stress distribution in the silicon element. Neglecting the shear components two effects occur: If current density and acting stress are parallel to each other the longitudinal effect (index I) is occurring, if current density and stress are acting perpendicularly the transversal effect (index $t$ ) is appearing. The interrelationship of resistance change and the described effects are combined in the following formula.

$$
\frac{\Delta R}{R_{0}} \approx \frac{\Delta \rho}{\rho}=\pi_{l} \cdot \sigma_{l}+\pi_{q} \cdot \sigma_{q}
$$

The $\pi$-coefficients (piezoresistive coefficients) depend on the material and its crystalline structure. To get the values for calculating the resulting resistance change and hence the sensitivity of the sensing element the manufacturing process has to be considered too. The manufacturing of the sensing elements is done by the Institute for Micro Sensors (CIS gGmbH) in Erfurt, Germany. In an established ion implanting process a boron concentration maximum of $N_{\mathrm{R}}=3^{*} 10^{18} \mathrm{~cm}^{-3}$ is adjusted to realise the piezoresistive areas. Thus, a surface resistivity of $R_{\square}=125 \Omega \mathrm{cm}$ results from the ion implanting. Due to the stress distribution in loading case and known implanting profile the resistance change can be calculated and $\pi$ coefficients can be evaluated. According to $[1,15]$ the longitudinal coefficient takes a value of $\Pi_{1}=$ $71.8^{*} 10^{-5} \mathrm{MPa}^{-1}$ and transversal coefficient $\pi_{\mathrm{q}}=-65.2^{*} 10^{-5} \mathrm{MPa}^{-1}$ for $<110>$-direction of silicon crystal.

Using equation 2 the sensitivity of the sensing element can be calculated from the simulated stress distribution. In FEA (Ansys) the stress distribution in the chip has been determined. Similarly to the analysis of the backlash on the bending behaviour several chip sizes have been simulated. The appropriate simulated setup is consistent with the setup shown in Fig. 1. Depending on the stress distribution the optimal position of the additional resistors can be defined. The position of the other resistors for measuring forces in $x$ direction can be similarly estimated. The resulting resistivity change caused by nominal force dependent on the analysed chip is shown in Fig. 4. Suitable positions of the piezoresistive areas are exemplarily marked in case of model No. 1 (see Fig. 4).

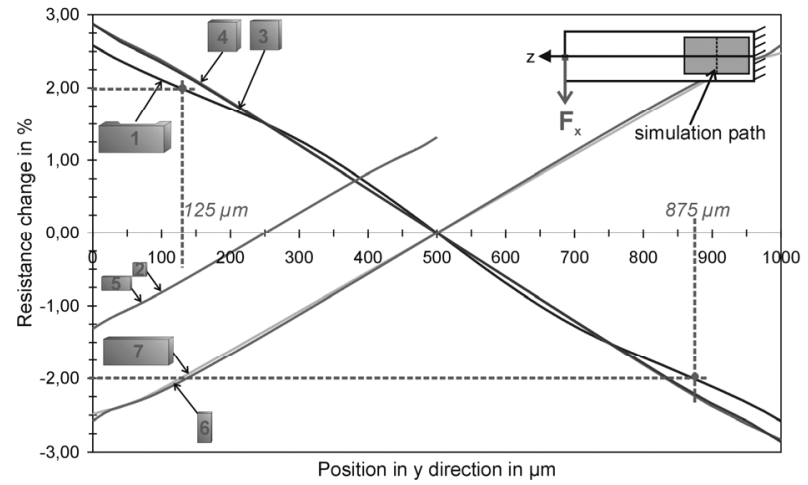

Fig. 4. Resistivity change of the analysed sensing elements

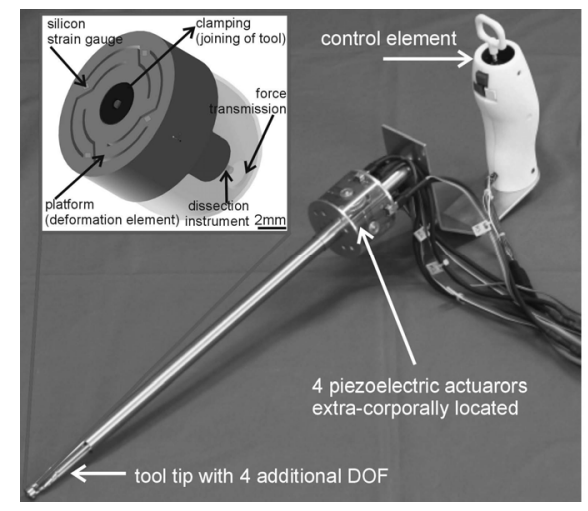

Fig. 5. Assembly of the realised instrument

\subsection{Assembly of the Force Sensor and Data Acquisition}

Having designed both sensing and deformation element the tri-axial force sensor can be built. Using a two-component epoxy adhesive ${ }^{8}$ one sensing element is bonded on each bending element. The contacting of the elements is done by wire bonding them to a flexible printed board. To protect wire bonds and Silicon chip a two component silicone ${ }^{9}$ is used. As shown in Fig. 5 the tool (laser-scalpel) is adapted to the deformation element. Since the laser-scalpel is a contactless dissection instrument the force transmission occurs at a special casing which ensures the required distance of $1 \mathrm{~mm}$ between tool tip and tissue. Dependent on the acting force component the strain distribution on each bending element and thus the stress distribution in each sensing element varies. To seperate the force components a linear system of equations shown in equation 3 has to be solved.

$$
\left(\begin{array}{l}
v_{1} \\
v_{2} \\
v_{3}
\end{array}\right)=\left(\begin{array}{lll}
a_{11} & a_{12} & a_{13} \\
a_{21} & a_{22} & a_{23} \\
a_{31} & a_{32} & a_{33}
\end{array}\right) \cdot\left(\begin{array}{l}
F_{x} \\
F_{y} \\
F_{z}
\end{array}\right)
$$

$F_{\mathrm{x}}, F_{\mathrm{y}}$ and $F_{\mathrm{z}}$ form the acting force vector, the matrix $A$ consisting of $a_{i j}$ has to be determind calibrating the sensor and $v_{i}$ are the resulting bridge voltages of the sensing element. After calculating the inverse matrix

\footnotetext{
${ }^{8}$ Loctite M-21HP

${ }^{9}$ Bayer Silicone, RT 601
} 
$A^{-1}$ the force vector can be determined. Due to the enduring manufacturing process of the sensing elements the behaviour of the tri-axial sensor is tested using conventional strain gauges.

\section{Conclusion}

A miniaturised force sensor with a high upper cut-off frequency for laparoscopic instruments has been developed. A two-component piezoresistive sensor has been manufactured and characterised, a piezoresistive tri-axial sensor is in progress. A functional modell using conventional strain gauges has been realised. Its characterisation under laboratory conditions will be accomplished in the next days, so that the measurement results can be presented at the conference in May 2009. Beside the characterisation of the sensor itself the co-action of the haptic force sensor and the active control has to be analysed. An other topic is to analyse the quality of the given haptic impression of the analysed tissue. In future the packaging of the sensing element and the assembly of the deformation element into the tool tip will be redesigned in order to meet the requirements of the Medical Device Directive. Also the influence of temperature gradients and humidity typically occuring during abdominal surgery has to be analysed.

\section{Acknowledgement}

The authors would like to thank $\mathrm{H}$. Bartuch, CIS gGMBH, fabricating the chips and P. Thiele, Aktiv Sensor $\mathrm{GmbH} / \mathrm{Epcos} \mathrm{AG}$, providing the pressure sensors. Dr. C. Hillert, UKE Hamburg, Dr. P. Hildebrand and Dr. R. Keller, UKL Lübeck, and Professor Dr. W. O. Bechstein, J. W. Goethe University Hospital Frankfurt (Main) are thanked for their assistance and advice too. Financial support was provided by the BMBF under grant 16SV2023.

\section{References}

[1] H. F. Schlaak, A. Roese, C. Wohlleber, S. Kassner, and R. Werthschützky. A Novel Laparoscopic Instrument with Multiple Degrees of Freedom and Intuitive Control. In European Biomedical Engineering Congress. Antwerpen, 2008.

[2] J. Rausch. Analyse der mechanischen Eigenschaften von Lebergewebe bei intrakorporaler Interaktion. Diploma thesis. TU Darmstadt, Institut EMK. 2006

[3] K. Emmermann, A. Kremer. Minimal-invasive Chirurgie: videolaparoskopische und videothorako-skopische Chirurgie. Stuttgart [u.a.]. 1995.

[4] R. Westebring-van der Putten, E. Goossens. Haptics in minimally invasive surgery - a review. Minimally Invasive Therapy and Allied Technologies, SMIT Journal, 17, 2008.

[5] P. Puangmali, K. Althoefer et al. State-of-the-Art in Force and Tactile Sensing for Minimally Invasive Surgery. IEEE Sensors Journal, 8(4). 2008.

[6] H. Mayer, I. Nágy, A. Knoll, E. Schirmbeck. An Experimental System for Robotic Heart Surgery. In 18th IEEE Symposium on Computer-Based Medical Systems (CBMS'05). 2005.

[7] J. Rosen, J. Brown, L. Chang, M. Barreca, M. Sinanan, and B. Hannaford. The BlueDRAGON - System for Measuring the Kinematics and the Dynamics of Minimally Invasive Surgical Tools In-Vivo. In International Conference on Robotics and Automation. Washington, May 2002.

[8] J. Peirs, J. Clijnen, D. Reynaerts, H. Van Brussel, P. Herijgers, B. Corteville, and S. Boone. A micro optical force sensor for force feedback during minimally invasive robotic surgery. Sensors and Actuators A: Physical, 115(23):447-455. 2004.

[9] P. Berkelman, L. Whitcomb, R. Taylor, P. Jensen. A miniature microsurgical Instrument Tip Force Sensor for Enhanced Force Feedback During Robot-Assisted Manipulation. Proceedings of IEEE Transaction on Robotics and Automation. 2003

[10] U. Seibold, B. Kuebler, H. Weiss, T. Ortmaier, and G. Hirzinger. Sensorized and Actuated Instruments for Minimally Invasive Robotic Surgery. In EuroHaptics. Munich, 2004.

[11] P. Valdastri, S. Roccella, L. Beccai, E. Cattin, A. Menciassi, M. Carrozza, and P. Dario. Characterization of a novel hybrid silicon threeaxial force sensor. Sensors and Actuators A Physical, 123-124:249-257. 2005.

[12] J. Rausch and R. Werthschützky. Development of Piezoresisitve Strain Gauges for Multi-Component Force Measurement in Minimally Invasive Surgery. In EuroSensors. Dresden, 2008.

[13] M.-H. Bao. Micro mechanical transducers: pressure sensors, accelerometers and gyroscopes, volume 8 of Handbook of sensors and actuators. Elsevier, Amsterdam [u.a.], 2 edition, 2004.

[14] S. Stavroulis. Rechnergestützter Entwurf von piezoresistiven Silizium-Drucksensoren mit realem mechanischem Wandler. PhD thesis. TU Darmstadt, Institut EMK. 2004.

[15] J. Mehner. Mechanische Beanspruchungsanalyse von Siliziumsensoren und -aktoren unter dem Einfluss von elektrostatischen und Temperaturfeldern. PhD thesis. TU Dresden. 1994.

[16] W. Schnell, D. Gross, and W. Hauger. Technische Mechanik 2: Elastostatik. Springer, Berlin [u.a.], 7 edition. 2002. 